\title{
The cold gas properties of Markarian galaxies
}

\author{
R. A. Kandalyan ${ }^{1,2,3, \star, \star \star}$ \\ 1 V. A. Ambartsumian Byurakan Astrophysical Observatory, 378433 Byurakan, Armenia \\ e-mail: rkandali@bao.sci.am \\ 2 Isaac Newton Institute of Chile, Armenian Branch, 378433 Byurakan, Armenia \\ 3 Institute of Astronomy and Space Sciences of the Al Al-Bayt University, PO Box 130040, Mafraq 25113, Jordan
}

Received 4 June 2002 / Accepted 25 October 2002

\begin{abstract}
A sample of 61 Markarian galaxies detected in the $\mathrm{CO}$ line was compiled. Using available $\mathrm{HI}_{2} \mathrm{H}_{2}$, optical and radio continuum data, the analysis of the gas kinematics and the star formation properties for this sample of galaxies was performed. The main conclusion can be summarized as follows:

(1) The HI and CO line widths are well correlated. Interaction between galaxies has no influence on the CO line broadening. A rapidly rotating nuclear disk in the galaxy might lead to the $\mathrm{CO}$ line broadening with less influence on the $\mathrm{HI}$ line.

(2) The atomic and molecular gas surface densities are well correlated with the blue, FIR and radio continuum surface brightness; however, the correlation for molecular component is stronger.

(3) In general, the galaxies with UV-excess (Markarian galaxies) do not differ in their star formation properties from the non-UV galaxies.
\end{abstract}

Key words. galaxies: ISM - radio lines: galaxies - ultraviolet: galaxies

\section{Introduction}

On the basis of luminosity alone, it is not possible to conclude whether or not star formation occurs in a burst. The FIR to blue luminosity ratio $L_{\mathrm{fir}} / L_{\mathrm{B}}$ provides a qualitative measure of the current star formation rate, and it may be strongly affected by extinction, while the gas emission in $\mathrm{mm}$ wavelengths is not affected by extinction.

It is well established that in luminous IR galaxies the star formation indicators, such as $L_{\mathrm{fir}} / L_{\mathrm{B}}$ or the flux density ratio at 60 and 100 microns $f 60 / f 100$ are better correlated with the molecular hydrogen than with the atomic hydrogen content (e.g. Young et al. 1989). The ratio $L_{\text {fir }} / M_{\mathrm{H}_{2}}$, where $M_{\mathrm{H}_{2}}$ denotes the molecular hydrogen mass, is usually interpreted as an indicator of efficiency of star formation (SFE) in galaxies. The SFE is independent of the Hubble type (Devereux \& Young 1991; Young et al. 1996) but depends on the environment of a galaxy (Sanders et al. 1991; Combes et al. 1994; Young et al. 1996). This dependence is more pronounced in interacting galaxies rather than in isolated ones. For the IR-bright galaxies, there is a linear relation between $\mathrm{H}_{2}$ and the dust content with SFE depending on the dust temperature of the warm component (Young et al. 1989). However, when longer wavelengths (mm range) were used in determining the dust

* e-mail: kandalyan@yahoo.com

$\star \star$ Present address: Institute of Astronomy and Space Sciences of the Al Al-Bayt University, PO Box 130040, Mafraq 25113, Jordan. content in IRAS-Mkn galaxies (Chini et al. 1992a), a strong correlation between $L_{\mathrm{fir}} / M_{\mathrm{gas}}$ and temperature of the cold dust was found. According to Andreani et al. (1995), the cold dust emission is associated with both the molecular and atomic hydrogen phases.

The CO line area is used to estimate the $\mathrm{H}_{2}$ gas mass of a galaxy. The line shape and line width of the $\mathrm{CO}$ emission involve information on distribution and kinematics of the gas (Krugel et al. 1990; Chini et al. 1992b).

Since molecular gas appears to play a critical role in the star formation process, it is of great importance to perform $\mathrm{CO}$ observations for galaxies, in particular for those with active starbursts.

Markarian galaxies exhibit a variety of activities, from starburst to nuclear, and this sample is one of the most suitable samples for investigation of the gas properties and the starburst phenomenon in galaxies. Using available $\mathrm{HI}, \mathrm{H}_{2}$, optical and radio continuum data, we have analyzed the gas kinematics and the star formation properties of $61 \mathrm{Mkn}$ galaxies. How do the gas properties of galaxies with UV- and non-UV-excess compare?

The value of the Hubble constant equal to $75 \mathrm{~km} \mathrm{~s}^{-1} \mathrm{Mpc}^{-1}$ is adopted throughout this paper. Section 2 presents a sample of 61 Markarian galaxies detected in the $\mathrm{CO}(1-0)$ line. In Sect. 3, the gas-luminosity relations are discussed. The results obtained are discussed in the final section. 
Table 1. Sample of $61 \mathrm{Mkn}$ galaxies.

\begin{tabular}{|c|c|c|c|c|c|c|c|c|c|c|c|c|c|c|c|}
\hline Mkn & $\begin{array}{c}i \\
\left({ }^{\circ}\right) \\
\end{array}$ & $\begin{array}{l}a_{\mathrm{o}} \\
\left({ }^{\prime}\right) \\
\end{array}$ & $\begin{array}{c}V \\
\mathrm{~km} \mathrm{~s}^{-1} \\
\end{array}$ & $\begin{array}{l}L_{\text {fir }} \\
L_{\odot} \\
\end{array}$ & $\begin{array}{c}L_{\mathrm{B}} \\
L_{\odot} \\
\end{array}$ & $\begin{array}{c}P_{\mathrm{R}} \\
\mathrm{W} \mathrm{Hz}^{-1} \\
\end{array}$ & $\begin{array}{l}M_{\mathrm{HI}} \\
M_{\odot} \\
\end{array}$ & $\begin{array}{c}M_{\mathrm{H} 2} \\
M_{\odot} \\
\end{array}$ & $\begin{array}{c}I_{\mathrm{CO}} \\
\mathrm{K} \mathrm{km} \mathrm{s}^{-1} \\
\end{array}$ & $\begin{array}{c}W H I \\
\mathrm{~km} \mathrm{~s}^{-1}\end{array}$ & $\begin{array}{r}W C O \\
\mathrm{~km} \mathrm{~s}^{-1} \\
\end{array}$ & $T$ & Env & Tel & Ref \\
\hline 2 & 32 & 0.73 & 5476 & 10.66 & 10.29 & & 9.45 & 9.39 & 7.5 & 167 & 106 & $\overline{0}$ & $\mathrm{p} ?$ & I & $\overline{1,2}$ \\
\hline 35 & 47 & 1.35 & 935 & 9.08 & 9.21 & & 8.00 & 7.60 & 1.0 & 96 & 41 & 3 & $\mathrm{i}$ & F & 3 \\
\hline 52 & 58 & 2.06 & 2252 & 9.80 & & & 8.70 & 8.73 & 2.3 & 96 & 60 & -1 & & F & 3 \\
\hline 88 & 0 & 0.19 & 9180 & 10.53 & 10.32 & 21.95 & 9.35 & 9.81 & 3.1 & 121 & 100 & 3 & $\mathrm{i}$ & $\mathrm{O}$ & 4 \\
\hline 91 & 0 & 0.89 & 5101 & 10.56 & 10.05 & & 9.40 & 9.58 & 6.0 & 201 & 113 & 3 & $\mathrm{i}$ & $\mathrm{O}$ & 4 \\
\hline 133 & 28 & 1.12 & 2010 & 9.58 & 9.81 & & 8.63 & 8.56 & 8.2 & 95 & 83 & 4 & & I & 1,2 \\
\hline 158 & 68 & 1.73 & 2070 & 9.99 & 10.01 & 21.04 & 8.67 & 9.07 & 25.3 & 209 & 126 & 1 & I & & 5 \\
\hline 171 & 47 & 1.89 & 3033 & 11.38 & 10.60 & 22.83 & & 9.78 & 60.0 & & 150 & 9 & ia & I & 6 \\
\hline 188 & 43 & 1.75 & 2404 & 9.98 & 10.20 & 21.41 & 8.91 & 9.16 & 22.8 & 270 & 240 & 5 & I & & 7 \\
\hline 201 & 61 & 1.61 & 2511 & 10.57 & 10.07 & 21.74 & 8.81 & 9.05 & 16.4 & 87 & 149 & 10 & & I & 7 \\
\hline 213 & 54 & 1.63 & 3115 & 10.04 & 10.29 & 21.36 & & 9.03 & 10.1 & & 311 & 1 & & I & 1,2 \\
\hline 231 & 43 & 1.22 & 12300 & 12.10 & 10.98 & 23.70 & & 10.42 & 16.0 & & 197 & 5 & ia & I & 7 \\
\hline 266 & 29 & 1.16 & 8358 & 11.18 & 10.69 & 22.82 & & 10.37 & 4.9 & & 400 & 7 & ia & $\mathrm{N}$ & 8 \\
\hline 273 & 90 & 0.81 & 11274 & 11.87 & 10.87 & 23.30 & & 10.44 & 19.8 & & 494 & 4 & ia & I & 7 \\
\hline 281 & 32 & 3.15 & 2227 & 9.96 & 10.42 & 21.07 & 8.86 & 9.19 & 6.8 & 305 & 278 & 3 & $\mathrm{i}$ & F & 9 \\
\hline 286 & 27 & 0.85 & 7548 & 10.86 & 10.62 & 21.76 & 10.17 & 9.88 & 5.4 & 231 & 175 & 4 & $\mathrm{i}$ & $\mathrm{O}$ & 4 \\
\hline 297 & 40 & 0.89 & 4701 & 10.64 & 10.38 & 22.36 & 9.47 & 9.26 & 12.5 & 366 & 180 & 5 & ia & NO & 10 \\
\hline 311 & 0 & 0.43 & 9190 & 10.70 & 10.37 & 21.86 & 9.12 & 9.60 & 1.9 & 194 & 124 & 6 & $\mathrm{i}$ & $\mathrm{O}$ & 4 \\
\hline 331 & 51 & 0.63 & 5351 & 11.11 & 10.07 & 22.19 & 9.93 & 10.06 & 16.1 & 264 & 281 & 3 & ia & $\mathrm{O}$ & 4 \\
\hline 332 & 24 & 1.42 & 2662 & 10.04 & 10.30 & 20.78 & 8.81 & 9.25 & 22.7 & 80 & 64 & 5 & & I & 5 \\
\hline 353 & 60 & 0.72 & 4861 & 10.43 & 10.25 & & 9.36 & 9.66 & 17.6 & 192 & 278 & 5 & $\mathrm{i}$ & I & 1,2 \\
\hline 363 & 59 & 3.82 & 2935 & 9.76 & & & 8.98 & 8.66 & 8.2 & 150 & & -2 & & NO & 11 \\
\hline 404 & & 0.10 & 1320 & 9.82 & & 21.25 & & 8.96 & 11.4 & & 280 & & & F & 3 \\
\hline 439 & 12 & 2.09 & 988 & 9.26 & 9.58 & 20.24 & 7.97 & 9.08 & 22.5 & 62 & 70 & 1 & & I & 12 \\
\hline 496 & 90 & 1.37 & 8785 & 11.16 & & 22.52 & 10.01 & 10.36 & 4.3 & 211 & 100 & -3 & ia & $\mathrm{N}$ & 8 \\
\hline 518 & 0 & 0.48 & 9506 & 10.85 & 10.69 & 22.36 & 9.75 & 10.05 & 5.0 & 242 & 199 & 10 & $\mathrm{i}$ & $\mathrm{O}$ & 4 \\
\hline 533 & 24 & 1.13 & 8662 & 11.08 & 10.85 & 23.10 & 10.00 & 10.31 & 4.0 & 449 & 145 & 4 & $\mathrm{p}$ & $\mathrm{N}$ & 8 \\
\hline 534 & 60 & 1.30 & 5119 & 10.72 & 10.60 & 22.14 & 9.50 & 9.75 & 8.7 & 286 & 420 & -2 & $\mathrm{p}$ & $\mathrm{O}$ & 12 \\
\hline 538 & 44 & 1.82 & 2801 & 10.35 & 10.26 & 22.05 & 9.02 & 9.03 & 12.4 & 163 & 177 & 3 & ia & I & 5 \\
\hline 545 & 52 & 1.87 & 4635 & 10.78 & 10.79 & 22.17 & 9.46 & 9.60 & 17.0 & 376 & 364 & 1 & $\mathrm{p}$ & I & 7 \\
\hline 575 & 40 & 0.81 & 5295 & 10.42 & 10.36 & 21.48 & 9.46 & 9.66 & 15.0 & 153 & 117 & 1 & i & I & 1,2 \\
\hline 602 & 44 & 1.32 & 2866 & 9.94 & 10.09 & 21.28 & 9.04 & 8.99 & 10.9 & 227 & 194 & 3.5 & & I & 1,2 \\
\hline 617 & 30 & 1.33 & 4723 & 11.27 & 10.44 & 22.48 & 9.39 & 9.56 & 14.8 & 250 & 255 & 5 & ia & I & 7 \\
\hline 620 & 44 & 3.46 & 1903 & 9.81 & 10.26 & 21.51 & 8.69 & 8.95 & 22.5 & 354 & 340 & 0.5 & & I & 7 \\
\hline 691 & 61 & 1.50 & 3297 & 10.14 & 10.36 & 21.62 & 9.14 & 9.00 & 8.4 & 143 & 100 & 4 & $\mathrm{p}$ & I & 1,2 \\
\hline 708 & 70 & 2.26 & 1897 & 9.74 & 9.85 & 21.11 & 8.65 & 9.03 & 27.1 & 242 & 197 & 5 & & I & 5 \\
\hline 731 & 49 & 2.39 & 1414 & 9.26 & & 20.41 & & 8.22 & 7.5 & & 100 & -1 & i & I & 1,2 \\
\hline 759 & 40 & 2.24 & 2066 & 9.76 & 10.16 & 21.82 & 8.78 & 8.70 & 10.7 & 208 & 167 & 5 & & I & 1,2 \\
\hline 769 & 64 & 1.98 & 1663 & 9.80 & 9.98 & 21.09 & 8.62 & 8.31 & 6.8 & 207 & 89 & 1 & & I & 5 \\
\hline 799 & 62 & 1.90 & 3028 & 10.48 & 10.39 & 21.77 & 9.53 & 9.70 & 49.8 & 314 & 309 & 3 & ia & I & 7 \\
\hline 848 & 60 & 0.82 & 12053 & 11.54 & & 22.83 & & 10.09 & 7.8 & & 93 & -2 & ia & I & 5 \\
\hline 928 & 42 & 1.14 & 7316 & 11.07 & & 22.65 & & 10.33 & 5.9 & & 259 & -1 & ia & $\mathrm{N}$ & 8 \\
\hline 938 & 74 & 1.75 & 5772 & 11.16 & & 22.51 & 9.66 & 9.78 & 16.7 & 418 & 347 & 3 & ia & I & 7 \\
\hline 1014 & & & 48893 & 12.14 & & 24.01 & & 10.70 & 0.2 & & 130 & & & $\mathrm{~N}$ & 13 \\
\hline 1034 & 0 & 0.43 & 10047 & 11.33 & 10.39 & 22.65 & 9.94 & 10.46 & 26.4 & 261 & 450 & 6 & & I & 7 \\
\hline 1040 & 90 & 2.87 & 4914 & 10.31 & 10.73 & & 9.59 & 9.47 & 1.8 & 440 & 500 & 4 & & $\mathrm{~N}$ & 14 \\
\hline 1050 & 54 & 1.10 & 4853 & 10.55 & 10.37 & 21.60 & 9.40 & 9.63 & 16.7 & 217 & 250 & 1 & $\mathrm{i}$ & I & 1,2 \\
\hline 1066 & 65 & 1.79 & 3605 & 10.56 & 10.29 & 22.02 & & 9.44 & 19.3 & & 271 & -1 & $\mathrm{p}$ ? & I & 7 \\
\hline 1073 & 21 & 1.04 & 6991 & 11.05 & 10.91 & 22.63 & 9.49 & 10.03 & 8.9 & 253 & 260 & 3 & $\mathrm{p}$ & $\mathrm{O}$ & 4 \\
\hline 1088 & 21 & 1.75 & 4626 & 10.61 & 10.64 & 22.02 & 9.37 & 9.50 & 13.4 & 297 & 377 & 0 & $\mathrm{i}$ & I & 7 \\
\hline 1093 & 48 & 1.10 & 4441 & 10.71 & & 22.05 & 9.92 & 9.74 & 6.1 & 359 & 253 & 1 & $\mathrm{p}$ ? & $\mathrm{S}$ & 5 \\
\hline 1157 & 36 & 1.28 & 4495 & 10.09 & & & 9.31 & 9.02 & 8.0 & 259 & 110 & 0 & & NO & 15 \\
\hline 1194 & 53 & 1.88 & 4552 & 10.64 & 10.63 & 21.85 & 9.36 & 9.96 & 40.2 & 269 & 291 & -2 & $\mathrm{i}$ & I & 7 \\
\hline 1259 & 44 & 2.00 & 2159 & 10.30 & & 21.53 & & 8.46 & 9.5 & & & -2 & & NO & 11 \\
\hline 1341 & 49 & 2.21 & 1132 & 9.09 & 9.57 & 20.14 & 8.23 & 7.95 & 6.4 & 186 & 155 & 6 & & I & 1,2 \\
\hline 1365 & 40 & 0.76 & 5652 & 10.55 & 10.16 & 21.83 & 9.44 & 9.59 & 11.2 & 180 & 217 & -2 & $\mathrm{i}$ & I & 1,2 \\
\hline 1376 & 90 & 2.90 & 1829 & 9.86 & 10.21 & 22.10 & 8.63 & 8.80 & 17.2 & 276 & 286 & 1 & & I & 5 \\
\hline 1379 & 58 & 1.46 & 2585 & 9.91 & 10.23 & 21.48 & 8.86 & 8.84 & 9.4 & 86 & 72 & 1.7 & & I & 1,2 \\
\hline 1405 & 26 & 1.37 & 4963 & 10.56 & 10.91 & & 9.48 & 9.83 & 11.1 & 281 & 280 & -3 & $\mathrm{p}$ ? & $\mathrm{O}$ & 4 \\
\hline 1466 & 43 & 4.38 & 1226 & 9.45 & 9.96 & 20.80 & 8.37 & 8.47 & 17.8 & 201 & 120 & 5 & & I & 7 \\
\hline 1485 & 45 & 3.14 & 2308 & 9.73 & 10.40 & & 8.91 & 8.79 & 10.5 & 286 & 240 & 3 & & I & 1,2 \\
\hline
\end{tabular}

1. Contini (1996); 2. Contini et al. (1997); 3. Young et al. (1995); 4. Kandalyan et al. (1998); 5. Chini et al. (1992b); 6. Solomon et al. (1992); 7. Krugel et al. (1990); 8. Sanders et al. (1991); 9. Jackson et al. (1989); 10. Sofue et al. (1993); 11. Taniguchi et al. (1991); 12. Wiklind \& Henkel (1989); 13. Sanders et al. (1988); 14. Heckman et al. (1989); 15. Taniguchi et al. (1990). 


\section{Results}

\subsection{A sample of Mkn galaxies detected in the CO line}

The flux limited ( $f 60<1.95 \mathrm{Jy}$ at 60 micron) sample of MknIRAS galaxies contains 155 objects (Kandalyan et al. 1995). In order to investigate the gas properties of Mkn-IRAS galaxies, we have extracted from the literature all the objects detected in the ${ }^{12} \mathrm{CO}(1-0)$ line (until May 2002). The total number of $\mathrm{CO}$ detected Mkn galaxies is 65. The galaxies Mkn 463, 551, 673 and 1027 have been detected in the CO line (Gao \& Solomon 1999), but the details of the line parameters, such as the CO line intensity and line width, are not presented. For this reason, we did not include these galaxies in our statistics. The optical, FIR and HI data have been extracted from the LEDA and NED ${ }^{1}$ databases. Additional HI data are chosen from Martin et al. (1991) and Kandalyan et al. (1997). The environment information on galaxies are taken from Mazzarella \& Balzano (1986); Mazzarella et al. (1991); Mazzarella \& Boroson (1993); Keel $\&$ van Soest (1992). The radio continuum data are chosen from Bicay et al. (1995) except those for Mkn 158, 188, 331, 404, 759, 1466 (Marx et al. 1994) and Mkn 213, 286, 439 (Stine 1992). For the environment and structure of a galaxy the following abbreviations are used: $\mathrm{p}$-pair of galaxies, i-isolated galaxy, ia-interactive galaxy. The notations: I-IRAM $30 \mathrm{~m}, \mathrm{~F}-$ FCRAO $14 \mathrm{~m}$, O-Onsala $20 \mathrm{~m}$, N-NRAO $12 \mathrm{~m}$, NO-NRA $45 \mathrm{~m}, \mathrm{~S}-\mathrm{SEST} 15 \mathrm{~m}$ for the radio telescopes are used. Table 1 presents the list of 61 galaxies and is arranged as follows:

Column 1: Markarian number of galaxy.

Column 2: Inclination angle in degrees $(i)$.

Column 3: Angular diameter $a_{\mathrm{o}}$ in arcmin.

Column 4: Heliocentric radial velocity $V$, in $\mathrm{km} \mathrm{s}^{-1}$.

Column 5: Logarithm of the FIR luminosity $L_{\mathrm{fir}}$, in solar units, calculated according to

$\log L_{\mathrm{fir}}=5.5954+2 \log D+\log (2.58 f 60+f 100)$, where $f 60$ and $f 100$ are the flux densities at 60 and 100 microns respectively, in Jy, and $D$ is the distance to the galaxy in Mpc.

Column 6: Logarithm of the blue luminosity, $L_{\mathrm{B}}$, in solar units, calculated according to $\log L_{\mathrm{B}}=12.164+2 \log D-0.4 B_{\text {To }}$, where $B_{\text {To }}$ is the total $B$ magnitude corrected for galactic and internal absorption.

Column 7: Logarithm of the radio continuum power $P_{\mathrm{R}}$ at $6 \mathrm{~cm}$, in $\mathrm{W} \mathrm{Hz}^{-1}$, calculated according to

$\log P_{\mathrm{R}}=17.078+2 \log D+\log F_{6}$, where $F_{6}$ is the flux density at $6 \mathrm{~cm}$ in $\mathrm{mJy}$.

Column 8: Logarithm of the atomic hydrogen mass, $M_{\mathrm{HI}}$, in solar mass, calculated according to

$\log M_{\mathrm{HI}}=5.372+\log I_{\mathrm{HI}}+2 \log D$, where $I_{\mathrm{HI}}$ is the integrated $\mathrm{HI}$ line intensity in $\mathrm{Jy} \mathrm{km} \mathrm{s}^{-1}$.

Column 9: Logarithm of the molecular hydrogen mass $M_{\mathrm{H}_{2}}$, in solar mass, which is calculated with use of the formula: $M_{\mathrm{H}_{2}}=4.78 L_{\mathrm{CO}}$, by taking the conversion factor from the $\mathrm{CO}$ emissivity into $\mathrm{H}_{2}$ column density as $N_{\mathrm{H}_{2}} / I_{\mathrm{CO}}=3 \times$ $10^{20} \mathrm{~cm}^{-2}\left(\mathrm{~K} \mathrm{~km} \mathrm{~s}^{-1}\right)^{-1}$ (Sanders et al. 1991). The CO line luminosity is calculated according to formula:

${ }^{1}$ The NASA-IPAC Extra-galactic Database (NED) which is operated by the Jet Propulsion Laboratory, Caltech under contract with the National Aeronautics and Space Administration (USA).
$L_{\mathrm{CO}}=26.6 D^{2}(\mathrm{Mpc}) \theta^{2}(\operatorname{arcsec}) I_{\mathrm{CO}}$, where $\theta$ is HPBW of the radio telescope.

Column 10: Integrated CO line intensity $I_{\mathrm{CO}}$ in $\mathrm{K} \mathrm{km} \mathrm{s}^{-1}$.

Column 11: HI line width $W H I$, in $\mathrm{km} \mathrm{s}^{-1}$, at half of the peak intensity.

Column 12: CO line width $W C O$, in $\mathrm{km} \mathrm{s}^{-1}$, at half of the peak intensity. The line widths are corrected for the galaxy inclination angle.

Column 13: Morphological type (T) as in RC3.

Column 14: Environment and structure of the galaxy.

Column 15: Radio telescope used for the CO line observation. Column 16: References for CO data. In the following discussion, the correlations were considered as real if the probability of random occurrence is less than 0.05 . The flux densities have not been corrected for redshift because all objects, except Mkn 1014, have redshifts much less than 0.1.

\subsection{The $\mathrm{HI}$ and $\mathrm{H}_{2}$ gas kinematics}

The gas kinematics of Mkn galaxies are studied by means of the statistical analysis of the $\mathrm{HI}$ and $\mathrm{CO}$ line widths. Figure 1 shows that there is a good correlation between WHI and WCO (correlation coefficient $r=0.72$ and its significance is $p<0.0001$ ). The relation presented in Fig. 1 indicates that the most part of the CO emission is likely to be co-planar with the largescale galaxy disk. The same result was obtained previously by Heckman et al. (1989) for a sample of Seyfert galaxies. The dispersion in Fig. 1 may be due to several causes. Firstly, the HI observations are usually carried out with a much larger beam width of the radio telescope than that of $\mathrm{CO}$ observations. Hence, the number of individual clouds belonging to the beam area is much higher for the HI observations than for $\mathrm{CO}$, so that the $\mathrm{HI}$ line width is a mean value from averaging over many clouds and the velocity dispersion among individual clouds may vary from one galaxy to another. Secondly, the dispersion in Fig. 1 could be also due to external and internal causes such as the environment of the galaxy and the starburst activity. It could be partly due to different behaviour of the gas rotation in galaxies. The least square fit of the $\mathrm{HI}$ and $\mathrm{CO}$ line widths data is

$W C O=(0.71 \pm 0.10) W H I+(59.4 \pm 41.8)$.

We believe that, due to the gas motion, different samples of galaxies show different slopes in the $\mathrm{HI}$ and $\mathrm{CO}$ line width relation.

In Table 2 we report mean values of $W H I, W C O$, their standard deviation and number for different types of galaxies. Sofue et al. (1993) and Tutui \& Sofue (1999) have suggested that tidal interaction could disturb the outermost but not the innermost regions of a galaxy. As a consequence, WHI for interacting galaxies will be much broader than that for isolated ones and no difference will be observed in WCO between the two types of galaxies. It can be seen from Table 2 that there are no significant differences between $W H I, W C O$ for isolated and paired+interacting galaxies, although for paired+interacting objects, WHI is slightly higher than for isolated galaxies $\left(458-377=81 \mathrm{~km} \mathrm{~s}^{-1}\right)$. It is noticeable that unclassified galaxies have smaller values of both WHI and WCO than those 
Table 2. Mean values of $W H I$ and $W C O$ for Mkn galaxies.

\begin{tabular}{lccrccc}
\hline \hline Env & $\begin{array}{c}W H I \\
\mathrm{~km} \mathrm{~s}^{-1}\end{array}$ & $S D$ & $N$ & $\begin{array}{c}W C O \\
\mathrm{~km} \mathrm{~s}^{-1}\end{array}$ & $S D$ & $N$ \\
\hline Isolated & 377 & 220 & 9 & 366 & 275 & 10 \\
Paired+ & 458 & 237 & 15 & 370 & 190 & 22 \\
interacting & & & & & & \\
Unclassified & 278 & 117 & 20 & 245 & 124 & 20 \\
All & 359 & 200 & 44 & 321 & 195 & 52 \\
\hline
\end{tabular}

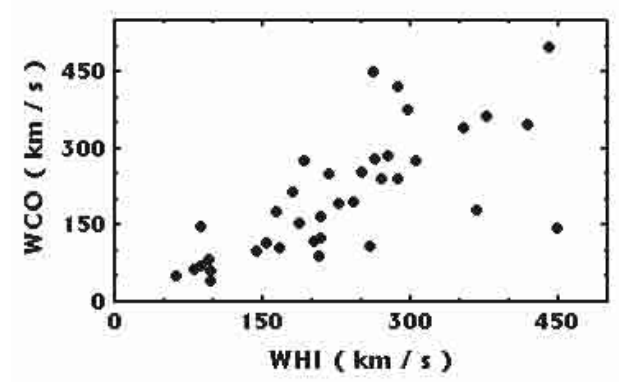

Fig. 1. The HI and CO line widths relation.

of isolated objects and significantly smaller than those for the paired+interacting galaxies. This fact is simply due to observational bias since, on the one hand, these objects are much fainter by global parameters such as $L_{\mathrm{B}}, L_{\mathrm{fir}}, M_{\mathrm{HI}}, M_{\mathrm{H}_{2}}$ (see Table 1) than classified galaxies, and, as a result, they have smaller line widths. On the other hand, because of the relative faintness of these objects, it is difficult to classify them. Nevertheless, when a part of unclassified galaxies were included in the group of isolated objects and another part in the group of paired+interacting galaxies, we still did not find significant differences for $W H I$ and $W C O$ between two main groups. Therefore interaction must have little influence on the $\mathrm{HI}$ line broadening and no influence on the $\mathrm{CO}$ line broadening in Mkn galaxies, although this problem needs a more detailed investigation based on a statistically significant and homogeneous sample.

The observed integrated $\mathrm{CO}$ line profiles in external galaxies result from the convolution of the antenna beam pattern with intrinsic emissivity distribution for which the velocity varies across the beam. The line profile contains information on the distribution and kinematics of the gas.

In general, the $\mathrm{HI}$ and $\mathrm{CO}$ lines are broadened by the velocity dispersion among individual clouds and/or by galactic rotation. Most of galaxies have an HI line width larger than that of $\mathrm{CO}$. The $\mathrm{CO}$ emission is generally concentrated within the central few kpc, while the HI gas distribution shows a depression in the central region of galaxy. The $\mathrm{CO}$ gas indicates the rotation and/or velocity dispersion among clouds in the innermost region including any rapidly rotating nuclear disk, whereas the HI gas indicates the rotation and velocity dispersion of the outer disk. Furthermore, superposition of individual clouds in the beam area is higher for HI observations than that for CO. Thus, in general, for a standard rotation curve (e.g. Sofue 1996, 1997, and the comprehensive review of Sofue \& Rubin 2001), we should expect an $\mathrm{HI}$ line width larger compared to the $\mathrm{CO}$ line width. Observationally, there exist galaxies with $F W H M$ of the CO line larger than that of the HI line (Kandalyan 1997; Tutui \& Sufue 1999). In these galaxies there may exist a rapidly rotating nuclear disk and/or expanding molecular gas due to the input of kinetic energy from supernovae and stellar winds associated with a starburst (e.g. NGC 1365, 4258). When the molecular gas in the central part of a galaxy has clumpy structure (Sakamoto et al. 1999; Regan et al. 2001), then the velocity dispersion among individual clouds will increase the line width. In the case of lack of the high velocity $\mathrm{HI}$ clouds in the central region of a galaxy, the FWHM of the CO line will be larger than that for the HI line. A bar or oval distortion could lead to $\mathrm{CO}$ line broadening and increased the star formation activity in a galaxy. Tutui \& Sofue (1999) argue that the CO line widths of the fast rotating galaxies tend to be larger than the HI line widths, while the HI line widths of slow rotating galaxies tend to be larger than the $\mathrm{CO}$ line widths.

Let us now discuss the difference between the $\mathrm{HI}$ and $\mathrm{CO}$ line widths. Figure 2 shows the histogram of (WHI-WCO) for Markarian galaxies. One can see that most of galaxies have $W H I>W C O$. However, according to Fig. 2, there exist galaxies with $W H I<W C O$. For the galaxies Mkn 201, $353,534,1034$, this difference is significant (higher than 0.01) and it is about 0.05 for Mkn 1088 and 1365. The inequality $W C O>W H I$ may indicate the existence of a rapidly rotating nuclear disk in the galaxy and, as a consequence, the rotation curves of these galaxies could have a peak in the central region $(<1 \mathrm{kpc})$, as in case of NGC 3031, 3079, 5236, 6946 (Sofue $1996,1997)$. The high angular resolution observations of the $\mathrm{HI}$ and $\mathrm{CO}$ are essential in testing this hypothesis.

The number of Mkn galaxies with a broad CO line width is insufficient for statistical analysis, but it is interesting that these galaxies are either barred or peculiar objects regardless of whether the galaxy is isolated, interacting or merging. Note that the velocity in barred spirals increases more steeply with radius than in unbarred ones. In the circumnuclear region of barred galaxies, the velocity field of the $\mathrm{CO}$ gas can have many different behaviours. For example, the velocity field in NGC 3504 is consistent with purely circular motion (Kenney et al. 1993), while in NGC 4314, both circular and non-circular motions have been observed (Benedict et al. 1996). The disk rotation curves of barred galaxies show dispersion larger than those of normal galaxies (Sofue et al. 1999). Recently Regan et al. (1999) have detected the high velocity (higher than circular velocity) streaming CO gas in seven barred galaxies. Objects with relatively broad $\mathrm{CO}$ emission will be very important for the study of the gas kinematics and dynamics of the central region.

\section{Gas content - luminosity relation}

\subsection{Surface densities versus blue surface brightness}

In the following discussion we will deal with quantities $S_{\mathrm{HI}}=$ $M_{\mathrm{HI}} / A, S_{\mathrm{H}_{2}}=M_{\mathrm{H}_{2}} / A, S_{\mathrm{fir}}=L_{\mathrm{fir}} / A, S_{\mathrm{B}}=L_{\mathrm{B}} / A$ and $S_{\mathrm{R}}=$ $P_{\mathrm{R}} / A$ normalized to surface area $A$ of a galaxy instead of $M_{\mathrm{HI}}$, $M_{\mathrm{H}_{2}}, L_{\mathrm{fir}}, L_{\mathrm{B}}$ and $P_{\mathrm{R}}$, in order to avoid the size effect. The blue luminosity is a tracer of the past star formation on the time scale of Gyr, whereas the FIR luminosity is a tracer of 
Table 3. Regression coefficients between surface densities and surface brightness. The slope $(S)$, correlation coefficient $(r)$ and probability $(p)$ that there is no correlation between the two variables are indicated in each box. The standard error is indicated in parentheses.

\begin{tabular}{lllll}
\hline \hline & $\log S_{\mathrm{B}}$ & $\log S_{\mathrm{fir}}$ & $\log S_{\mathrm{R}}$ & $\log S_{\mathrm{HI}}$ \\
\hline $\log S_{\mathrm{HI}}$ & $S=0.97(0.18)$ & $S=0.45(0.06)$ & $S=0.28(0.08)$ & \\
(all) & $r=0.64(0.09)$ & $r=0.76(0.08)$ & $r=0.51(0.12)$ & \\
& $p<3 \times 10^{-6}$ & $p<10^{-6}$ & $p<0.002$ & \\
\hline $\log S_{\mathrm{HI}}$ & $S=0.95(0.29)$ & $S=0.65(0.21)$ & $S=0.39(0.26)$ & \\
(isolated) & $r=0.70(0.19)$ & $r=0.69(0.23)$ & $r=0.50(0.35)$ & \\
& $p<0.01$ & $p<0.01$ & $p=0.17$ & \\
\hline $\log S_{\mathrm{HI}}$ & $S=0.35(0.59)$ & $S=0.57(0.21)$ & $S=0.48(0.26)$ & \\
(paired+ & $r=0.18(0.31)$ & $r=0.65(0.22)$ & $r=0.50(0.27)$ & \\
interacting) & $p=0.59$ & $p<0.02$ & $p=0.09$ & \\
\hline $\log S_{\mathrm{H} 2}$ & $S=1.33(0.31)$ & $S=0.73(0.09)$ & $S=0.57(0.10)$ & $S=1.06(0.14)$ \\
(all) & $r=0.56(0.11)$ & $r=0.78(0.08)$ & $r=0.65(0.10)$ & $r=0.77(0.10)$ \\
& $p<10^{-4}$ & $p<10^{-6}$ & $p<10^{-6}$ & $p<10^{-6}$ \\
\hline $\log S_{\mathrm{H} 2}$ & $S=1.23(0.35)$ & $S=0.64(0.22)$ & $S=0.50(0.21)$ & $S=0.87(0.22)$ \\
(isolated) & $r=0.73(0.19)$ & $r=0.68(0.19)$ & $r=0.64(0.23)$ & $r=0.77(0.18)$ \\
& $p<0.005$ & $p<0.01$ & $p=0.04$ & $p<0.002$ \\
\hline $\log S_{\mathrm{H}_{2}}$ & $S=0.33(0.53)$ & $S=0.48(0.21)$ & $S=0.36(0.18)$ & $S=0.62(0.17)$ \\
(paired+ & $r=0.20(0.28)$ & $r=0.58(0.24)$ & $r=0.43(0.21)$ & $r=0.75(0.19)$ \\
interacting) & $p=0.54$ & $p<0.05$ & $p=0.06$ & $p<5 \times 10^{-3}$ \\
\hline
\end{tabular}

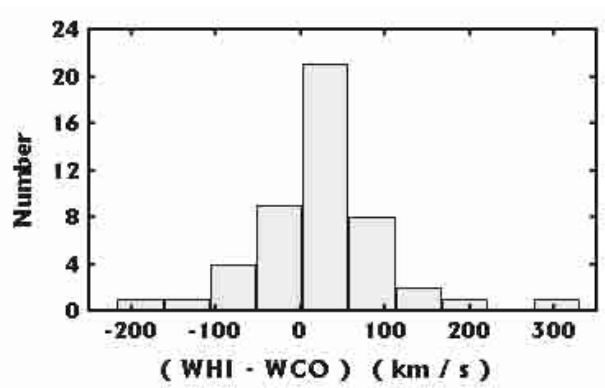

Fig. 2. Distribution of the $\mathrm{HI}$ and $\mathrm{CO}$ line widths difference.

the recent star formation on the time scale of Myr. We made use of multiple regression analysis to correct the distance effect (Malmquist bias). The corrected regression coefficients are presented in Table 3. In order to have a sufficient number of objects in each morphological group for statistics, we have included paired and interacting galaxies in one group. From Table 3, one can see that the $\mathrm{HI}$ and $\mathrm{H}_{2}$ surface densities are correlated with the blue surface brightness for isolated galaxies only, and correlation between these quantities for the whole sample is completely due to isolated galaxies. We can conclude that, for isolated galaxies, both $S_{\mathrm{HI}}$ and $S_{\mathrm{H}_{2}}$ depend linearly on the past star formation activity.

\subsection{Surface densities versus surface FIR brightness}

Now we discuss the relationships between the gas surface densities and the FIR surface brightness. The corrected regression coefficients are presented in Table 3. The FIR surface brightness is well correlated with the $\mathrm{HI}$ and $\mathrm{H}_{2}$ surface densities for both isolated and paired+interacting galaxies. This suggests that, with an increase in the gas surface density, the FIR surface brightness increases irrespective of the galaxy environment. Another interesting result is the non-linearity of the relation between the HI gas surface density and the FIR surface brightness. This is contrasted with the almost linear relation between the $\mathrm{H}_{2}$ gas surface density and the FIR surface brightness. This indicates that the recent star formation activity exhibits stronger dependence on the $\mathrm{H}_{2}$ gas phase than on the $\mathrm{HI}$ phase. Furthermore, recently, Wong \& Blitz (2002) used the azimuthally-averaged data for seven CO-bright spiral galaxies and found that the SFR surface density exhibits a much stronger correlation with the $\mathrm{H}_{2}$ gas surface density than with the HI gas surface density. Moreover, there exists a quasi-linear relation between the SFR and $\mathrm{H}_{2}$ surface densities. Therefore the star-forming gas in these seven galaxies exists predominantly in the molecular form. It should be noted that the mean values of $L_{\mathrm{fir}}, L_{\mathrm{B}}, M_{\mathrm{HI}}$ and $M_{\mathrm{H}_{2}}$ do not differ significantly for isolated and paired+interacting Mkn galaxies.

\subsection{Surface densities versus radio continuum surface brightness}

It is well known that the linear relation between the radio continuum and IR emission in galaxies is a consequence of the star formation activity. It is assumed that the cosmic rays arise together with ionizing radiation during the star formation. The ionized gas emits thermal radio emission, while the cosmic ray electrons interacting with a magnetic field emit synchrotron radio emission. Thus, the radio continuum and IR emission are due to the star formation activity. On the other hand, the star formation activity is related to the gas content of a galaxy. Therefore, the study of the relationship between the radio continuum emission and the gas content of galaxies is of interest. The regression coefficients corrected for the distance effect are reported in Table 3. One can see that both phases of the gas are related to the radio continuum surface brightness, but $S_{\mathrm{R}}$ is related to $S_{\mathrm{H}_{2}}$ more strongly than to $S_{\mathrm{HI}}$. When isolated and paired+interacting galaxies are considered separately, the confidence levels of the relationships are not very high. However, 
it could be due to the small number of objects in each group, especially for the group of isolated galaxies ( 11 objects). Thus, the radio continuum emission in Markarian galaxies is related to both phases of the gas, but the relation to the molecular gas is much stronger than to the atomic one. In general, the results of Sect. 3 are in good agreement with those of Casoli et al. (1996).

\section{Discussion}

A great number of articles have been devoted to investigation of the gas properties of galaxies (e.g., review by Young \& Scoville 1991). In particular, gas-to-luminosity relations of various types of galaxies have been studied extensively. The results of comparison between the gas content and luminosity reveal different effects. For example, in the FIR luminous objects $\left(\log L_{\mathrm{fir}} \gtrsim 10.5\right)$, the HI content is not correlated with the FIR luminosity, while for fainter objects, there exist a strong correlation between these quantities (Kandalyan et al. 1997). In nearby spirals, the $\mathrm{HI}$ content is unrelated to the $\mathrm{H}_{2}$ content (Braine \& Combes 1992), whereas in the IRAS-selected galaxies there is a strong correlation between them (see, e.g., present section; Young et al. 1989; Andreani et al. 1995). Thus, gas phase to gas phase and gas phase to luminosity relations in the galaxies are different and very complicated and depend on many internal and external factors such as the environment, luminosity, morphology, star formation history etc.

Up to now, the atomic and molecular hydrogen properties have been investigated on the basis of either optically- or IRASselected samples of galaxies. The different samples reveal different gas-to-luminosity relations in the galaxies. For instance, IRAS-selected samples are biased toward galaxies with recent or present star forming activity, while in the optically-selected samples, the past star forming galaxies dominate. In Table 4 we summarized the main results of the comparison of the $\mathrm{HI}, \mathrm{H}_{2}$ surface densities and surface brightness obtained from various samples of galaxies, where $B, F I R$ and $R$ denote the blue, farinfrared and radio continuum surface brightness, respectively. The first line in each box corresponds to $S_{\mathrm{HI}}$, and the second line to $S_{\mathrm{H}_{2}}$. The presence of either HI or $\mathrm{H}_{2}$ indicates the existence of a correlation between the surface density and corresponding surface brightness. " $n$ " indicates the absence of a correlation between two quantities. An empty field means that the relation has not been considered. Bold letters indicate that the considered correlation is stronger than the relationship between the two other variables in the same box. When several articles were available in the literature for each sample, the results were combined and in the references the most recent papers are presented. Table 4 includes only five samples which, in our opinion, represent the main samples studied for the $\mathrm{CO}$ emission so far. We have used the following abbreviations: "Nearby" - optically selected nearby galaxies; "Starburst" optically selected starburst galaxies; "UV-IRAS" - Markarian galaxies detected by IRAS; "IRAS" - IRAS selected galaxies; "Cluster" - clusters' galaxies. These samples are comprised mainly of spiral galaxies. Several important conclusions can be drawn from Table 4. (a) For all samples, the FIR surface brightness is correlated with the molecular hydrogen surface density more strongly than with the neutral hydrogen surface density.
Table 4. Comparison of star formation activities of different samples.

\begin{tabular}{lllllr}
\hline \hline Sample & $B$ & $F I R$ & $R$ & $S_{\mathrm{HI}}$ & Reference \\
\hline Nearby & HI & HI & $\mathrm{n}$ & & $1,2,3$ \\
& H2 & H2 & H2 & $\mathrm{n}$ & \\
\hline Starburst & HI & HI & & & 4 \\
& H2 & H2 & & H2 & \\
\hline UV-IRAS & HI & HI & HI & & 5 \\
& H2 & H2 & H2 & H2 & \\
\hline IRAS & HI & HI & & & $6,7,8$ \\
& H2 & H2 & & H2 & \\
\hline Cluster & HI & HI & $\mathrm{n}$ & & 9 \\
& H2 & H2 & H2 & n & \\
\hline
\end{tabular}

1. Braine \& Combes (1992); 2. Sage (1993); 3. Elfhag et al. (1996); 4. Jackson et al. (1989); 5. Present work; 6. Young et al. (1989); 7. Sanders et al. (1991); 8. Andreani et al. (1995); 9. Casoli et al. (1996).

This suggests that, during present or recent star formation, the molecular phase of the gas plays an immediate part in the star formation.

(b) The strong correlation between the HI surface density and the FIR surface brightness for all samples indicates that, in present or recent star formation, the neutral hydrogen phase is also important. Of course, in some galaxies, a part of the FIR emission could originate in the diffuse atomic medium and not be related to the star formation regions. Thus, we can conclude that both phases of the gas are important for star formation. Casoli et al. (1996) arrived at the same conclusion.

(c) It is clear from Table 4 that both phases of the gas are related to the past star forming indicator $S_{\mathrm{B}}$. But now the molecular phase does not dominate in this relation for all samples, as it was in the case of $S_{\text {fir }}$. Only in the "UV-IRAS" and "IRAS" samples did $S_{\mathrm{B}}$ correlate with $S_{\mathrm{H}_{2}}$ more strongly than with $S_{\mathrm{HI}}$. Hence, both components of the gas are important for the past star formation activity.

(d) Unfortunately, the radio continuum data were not available for all samples of Table 4. Nevertheless one can see that there is a strong correlation between $S_{\mathrm{R}}$ and $S_{\mathrm{H}_{2}}$. In the "UV-IRAS" sample, the radio continuum surface brightness is correlated also with the atomic hydrogen surface density. The universal correlation between the radio continuum and FIR emission, which is observed from normal galaxies to quasars, is due to the star formation activity (e.g. Kandalyan 1996). Therefore correlation between $S_{\mathrm{R}}$ and $S_{\mathrm{H}_{2}}$ has the same origin as the former relation. From this point of view the observed relation between $S_{\mathrm{R}}$ and $S_{\mathrm{HI}}$ for the "UV-IRAS" sample may indicate that the FIR and HI emissions in these objects are basically due to the star formation while in the "Nearby" and "Cluster" samples a part of the FIR and HI emissions could be related to the diffuse interstellar medium.

(e) It is very difficult to understand the absence of correlation between $S_{\mathrm{HI}}$ and $S_{\mathrm{H}_{2}}$ for "Nearby" and "Cluster" samples. In fact, if we believe that both components of the gas are important for star formation, we expect to observe a relationship between them. Perhaps for some samples this relationship is too weak to be observed. But the absence of correlation definitely 
does not depend on the environment of a galaxy (field or cluster objects).

(f) Table 4 shows that Markarian galaxies do not differ in star formation properties from other galaxies. The resemblance of "Nearby" and "Cluster" samples is noticeable.

\section{Conclusions}

The main results of this work may be summarized as follows: 1. A sample of $61 \mathrm{Mkn}$ galaxies detected in the $\mathrm{CO}(1-0)$ line was compiled for investigation of the star formation activity. These galaxies were selected from the complete sample of Markarian objects detected by IRAS. The HI, $\mathrm{H}_{2}$, optical and radio continuum data available from the literature are presented for 61 galaxies.

2. The HI and CO line widths are well correlated. Although the $\mathrm{HI}$ line width for interacting objects is slightly larger than that for isolated galaxies, it is proposed that interaction has little influence on the HI line broadening in Markarian galaxies. The galaxy interaction has no influence on the CO line broadening. 3 . It was suggested that the rapidly rotating nuclear disk in a galaxy could lead to a $\mathrm{CO}$ line width greater than the HI line width.

4. The $\mathrm{HI}$ and $\mathrm{H}_{2}$ gas phases are well correlated with both the past and present star formation indicators such as the blue, FIR and radio continuum surface brightness. However, the molecular phase is related to these indicators more strongly than to the atomic phase. The gas phases are also correlated. In some relations, the isolated and interacting galaxies have different behaviour (see Table 3 ).

5. In general, the galaxies with UV-excess (Markarian galaxies) do not differ in their star formation properties from the non-UV galaxies.

Acknowledgements. I gratefully thank J.-M. Martin, for his consent to use some unpublished results of observations. It is a great pleasure to thank A. Nikoghossian for the valuable comments. I wish to thank the anonymous referee who helped improve the manuscript. This research has made use of the NASA-IPAC Extragalactic Database (NED) which is operated by the Jet Propulsion Laboratory, Caltech, under contract with the National Aeronautics and Space Administration (USA); the Lyon-Meudon Extragalactic Database (LEDA), supplied by the LEDA team at CRAL-Observatoire de Lyon (France).

\section{References}

Andreani, P., Casoli, F., \& Gerin, M. 1995, A\&A, 300, 43

Benedict, F. G., Smith, B. J., \& Kenney, J. D. P. 1996, AJ, 111, 1861

Bicay, M. D., Kojoian, G., Seal, J., Dickinson, D. F., \& Malkan, M. A. 1995, ApJS, 98, 369

Braine, J., \& Combes, F. 1992, A\&A, 264, 433

Casoli, F., Dickey, J., KazŁs, I., Boselli, A., Gavazzi, P., \& Baumgardt, K. 1996, A\&A, 309, 43

Chini, R., Krugel, E., \& Kreysa, E. 1992a, A\&A, 266, 177

Chini, R., Krugel, E., \& Steppe, H. 1992b, A\&A, 255, 87

Combes, F., Prugniel, P., Rampazzo, R., \& Sulentic, J. W. 1994, A\&A, 281,725

Contini, T. 1996, Ph.D. Thesis, Universite Paul Sabatier, Toulouse, France
Contini, T., Wozniak, H., Considere, S., \& Davoust, E. 1997, A\&A, 318, L51

Devereux, N. A., \& Young, J. S. 1991, ApJ, 371, 515

Elfhag, T., Booth, R. S., Hoglund, B., Johansson, L. E. B., \& Sandqvist, Aa. 1996, A\&AS, 115, 439

Gao, Yu, \& Solomon, P. M. 1999, ApJ, 512, L99

Heckman, T. M., Blitz, L., Wilson, A. S., \& Armus, L. 1989, ApJ, 342, 735

Jackson, J. M., Snell, R. L., Ho, P. T. P., \& Barrett, A. H. 1989, ApJ, 337,680

Kandalyan, R. A. 1996, Afz, 39, 337

Kandalyan, R. A. 1997, Galaxy Interaction at Low and High Redshift, IAU Symp. 186, ed. J. Barnes, \& D. B. Sanders (Kluwer Academic Publishers), 84

Kandalyan, R. A., Martin, J.-M., Bottinelli, L., \& Gouguenheim, L. 1995, Afz, 38, 639

Kandalyan, R. A., Martin, J.-M., Bottinelli, L., \& Gouguenheim, L. 1997, unpublished

Kandalyan, R. A., Martin, J.-M., Horellou, C., Bottinelli, L., \& Gouguenheim, L. 1998, unpublished

Keel, W. C., \& van Soest, E. T. M. 1992, A\&AS, 94, 553

Kenney, J. D. P., Carlstrom, J. E., \& Young, J. S. 1993, ApJ, 418, 687

Krugel, E., Steppe, H., \& Chini, R. 1990, A\&A, 229, 17

Martin, J.-M., Bottinelli, L., Dennefeld, M., \& Gouguenheim, L. 1991, A\&A, 245, 393

Marx, M., Krugel, E., Klein, U., \& Wielebinski, R. 1994, A\&A, 281, 718

Mazzarella, J. M., \& Balzano, V. A. 1986, ApJS, 62, 751

Mazzarella, J. M., \& Boroson, T. A. 1993, ApJS, 85, 27

Mazzarella, J. M., Bothun, G. D., \& Boroson, T. A. 1991, AJ, 101, 2034

Regan, M. W., Sheth, K., \& Vogel, S. N. 1999, ApJ, 526, 97

Regan, M. W., Thornly, M. D., Helfer, T. T., et al. 2001, ApJ, 561, 218

Sage, L. J. 1993, A\&A, 272, 123

Sakamoto, K., Okumura, S. K., Ishizuki, S., \& Scoville, N. Z. 1999, ApJS, 124, 403

Sanders, D. B., Scoville, N. Z., \& Soifer, B. T. 1988, ApJ, 335, L1

Sanders, D. B., Scoville, N. Z., \& Soifer, B. T. 1991, ApJ, 370, 158

Sofue, Y. 1996, ApJ, 458, 120

Sofue, Y. 1997, PASJ, 49, 17

Sofue, Y., \& Rubin, V. 2001, ARA\&A, 39, 137

Sofue, Y., Tutui, Y., Honma, M., et al. 1999, ApJ, 523,136

Sofue, Y., Wakamatsu, K., Taniguchi, Y., \& Nakai, N. 1993, PASJ, 45, 43

Solomon, P. M., Downes, D., \& Radford, S. J. E. 1992, ApJ, 387, L55

Stine, P. C. 1992, ApJS, 81, 49

Taniguchi, Y., Kameya, O., Nakai, N., \& Kawara, K. 1990, ApJ, 358, 132

Taniguchi, Y., Kameya, O., \& Nakai, N. 1991, Dynamics of Galaxies and Their Molecular Distribution, ed. F. Combes, \& F. Casoli (Kluwer Academic Publishers), IAU Symp., 146, 328

Tutui, Y., \& Sufue, Y. 1999, A\&A, 351, 467

Wiklind, T., \& Henkel, C. 1989, A\&A, 225, 1

Wong, T., \& Blitz, L. 2002, ApJ, 569, 157

Young, J. S., \& Scoville, N. Z. 1991, ARA\&A, 29, 581

Young, J. S., Lori, A., Kenney, J. D. P., Lesser, A., \& Rownd, B. 1996, AJ, 112, 1903

Young, J. S., Xie, S., Kenney, J. D. P., \& Rice, W. L. 1989, ApJS, 70, 699

Young, J. S., Xie, S., Tacconi, L., et al. 1995, ApJS, 98, 219 